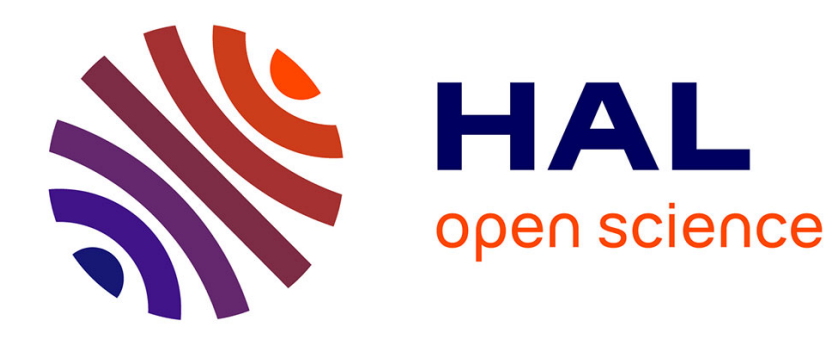

\title{
N'importe quoi ! Le hors-sujet de l'énonciation
}

Dominique Ducard

\section{To cite this version:}

Dominique Ducard. N'importe quoi! Le hors-sujet de l'énonciation. La prise en charge énonciative. Études théoriques et empiriques, Bruxelles, De Boeck-Duculot, pp.183-198, 2011. halshs-01146609

\section{HAL Id: halshs-01146609 \\ https://shs.hal.science/halshs-01146609}

Submitted on 10 Nov 2020

HAL is a multi-disciplinary open access archive for the deposit and dissemination of scientific research documents, whether they are published or not. The documents may come from teaching and research institutions in France or abroad, or from public or private research centers.
L'archive ouverte pluridisciplinaire HAL, est destinée au dépôt et à la diffusion de documents scientifiques de niveau recherche, publiés ou non, émanant des établissements d'enseignement et de recherche français ou étrangers, des laboratoires publics ou privés. 


\title{
N'IMPORTE QUOI ! LE HORS-SUJET DE L'ÉNONCIATION
}

\author{
Dominique Ducard \\ in Patrick Dendale et al., La prise en charge énonciative
}

De Boeck Supérieur | « Champs linguistiques »

2011 | pages 183 à 198

ISBN 9782801116432

Article disponible en ligne à l'adresse :

https://www.cairn.info/prise-en-charge-enonciative---page-183.htm

Distribution électronique Cairn.info pour De Boeck Supérieur.

(C) De Boeck Supérieur. Tous droits réservés pour tous pays.

La reproduction ou représentation de cet article, notamment par photocopie, n'est autorisée que dans les limites des conditions générales d'utilisation du site ou, le cas échéant, des conditions générales de la licence souscrite par votre établissement. Toute autre reproduction ou représentation, en tout ou partie, sous quelque forme et de quelque manière que ce soit, est interdite sauf accord préalable et écrit de l'éditeur, en dehors des cas prévus par la législation en vigueur en France. Il est précisé que son stockage dans une base de données est également interdit. 


\title{
N'IMPORTE QUOI! LE HORS-SUJET DE L'ÉNONCIATION
}

\author{
Dominique DUCARD ${ }^{1}$
}

\section{Avant de commencer}

Je dois préciser, en préalable, que si je vais me placer dans le sillage de la théorie des opérations énonciatives d'A. Culioli et que je suivrai de près certaines des études de ce linguiste, je me tiens plutôt en bordure de cette linguistique de l'énonciation, dans une perspective sémiolinguistique d'analyse des paroles et des discours dont je dirai un mot à la fin.

Un autre préalable s'impose quant à la terminologie utilisée en référence à la notion de prise en charge. A. Culioli emploie cette notion, le premier semble-t-il et après une discussion sur ce point avec Robert Martin², dès 1970 dans l'un des articles de linguistique qu'il a rédigés pour une encyclopédie généraliste (Culioli 1970), à propos de la modalité: « Toute énonciation suppose une prise en charge par un énonciateur», et il renvoie à l'article assertion. Un peu plus loin il précise que «tout acte d'énonciation suppose une attitude prise à l'égard de la relation que contient la lexis» (relation prédicative) et insiste en ajoutant ensuite :

1. Université Paris Est-Créteil - Céditec (EA 3119).

2. Communication personnelle d'A. Culioli. 
«Il n'existe pas d'énonciation sans modalisation, mais la modalité appartient à plusieurs domaines que l'on sépare de façon plus ou moins arbitraire (cognitif / affectif, jugements, relations entre sujets énonciateurs, etc.)» (Culioli 1970).

Le séminaire de 1983-1984 (Culioli 1985) reprend cette question quand il s'agit d'établir la distinction essentielle entre locuteur et énonciateur, qui sont deux instances séparables:

«pour produire une assertion, il faut déclarer publiquement [...]; et il faut d'un autre côté qu'il y ait un engagement d'une personne qui prend en charge, qui se porte garante, qui tient à affirmer quelque chose envers et contre tout. Si vous avez simplement instance de locution, vous n'avez pas véritablement assertion. Pour avoir prise en charge, il faut se porter garant; et si c'est à l'intérieur d'un cadre institutionnel, le garant peut être sanctionné si ce dont il se porte garant ne se réalise pas.»

Le terme engagement sera ensuite préféré au syntagme prise en charge, qui est alors rapporté à un acte de responsabilité appréhendé par ses conséquences et dont la validité peut être envisagée en raison d'une sanction éventuelle. L'engagement implique une prise de position de la part d'un énonciateur mobile ${ }^{3}$ et comprend aussi bien l'engagement que le non-engagement ou le désengagement, et même le ré-engagement. Il faudrait à ce titre écrire le terme en capitales: ENGAGEMENT. De plus, la prise en charge ne se conçoit guère avec un gradient, sauf à y introduire une mesure de pondération selon le poids de la charge et l'effort du porteur, comme nous y invitent les significations associées à la notion, alors que l'engagement, comme nous le verrons, va de pair avec une valeur d'intensité.

L'étude de cas que je présente vise à éclaircir cette notion métalinguistique, dans le cadre de la théorie des opérations énonciatives, et de montrer comment elle a conduit, dans les travaux plus récents d'A. Culioli, à la théorisation de la force assertive, qui n'est pas un désaveu de la notion de «prise en charge », mais un dépassement par un déplacement tenant compte de l'extension et de la variété des faits linguistiques à traiter, avec une conception moins figée de l'intersubjectivité dans l'activité de langage.

\section{Pour commencer}

Dans plusieurs études j'ai utilisé le débat parlementaire, à l'Assemblée nationale ou au Sénat, comme un observatoire de l'espace de confrontation de la parole, propice à l'examen de la dimension à la fois intersubjective et impersonnelle de l'activité énonciative. Cet observatoire, d'où je tire quelques-unes des multiples occurrences de la forme qui m'intéresse, me servira ici seulement de toile de fond.

3. C'est-à-dire qui peut changer de position. 
Il s'agit en effet, au cours de ces débats, non seulement d'affirmer des croyances et des convictions, celles qui déterminent des choix et guident l'action, mais aussi de les fonder sur ce qu'est le vrai, le juste, le bon, l'utile, de façon circonstanciée et contingente ou au nom d'idéaux supra-individuels. Le déroulement des discours, qui fait alterner les interventions présentant et discutant les questions à l' ordre du jour, est ponctué par les réactions des participants qui, en tant que membres du chœur des partisans ou des opposants, approuvent, encouragent, se félicitent, se réjouissent ou démentent, attaquent et contre-attaquent, s'indignent, se désolent, etc. Je retiendrai de ces interventions sur ce qui se dit la valeur d'assentiment ou de consentement - selon l'acception classique d'adhésion à des idées et de sentiment partagé - ou, à l'inverse, de dissentiment et, à un degré plus fort dans l'expression de la division, de dissension, sous-tendue par une certaine éthique de la parole, comme le montrent les accusations de mensonge, de tromperie, de mauvaise foi, de dissimulation, de travestissement, etc. (Voir par ex. les deux items 3 et 4 du Doc. $C$ en annexe). Les questions qui animent, vivement, les échanges portent alors sur ce qu'est dire - ou ne pas dire ou ne pas dire tout à fait - le vrai sur le vrai, et ce qui engage la relation des interlocuteurs au monde et aux autres. À titre d'exemples je donnerai, en vrac, ce relevé des exclamations qui ponctuent les discours des orateurs et qui relèvent toutes, au-delà de la particularité de chaque forme et de son interprétation, d'une évaluation du propos tenu et, plus précisément, de la validation (ou invalidation) d'une assertion: «Très bien!, Ce n'est pas vrai !, Ça, c'est vrai !, Ce n'est pas crédible !, Bien sûr!, Tout à fait!, Très bien!, Il fallait le dire !, Très juste !, C'est sûr !, C'est le moins qu'on puisse dire!,C'est fallacieux !,C'est faux !, Mais non !,Exactement !,C'est le contraire !, Absolument!, C'est juste !, Parfaitement!, Pas du tout !, C'est la vérité !, C'est évident!, Vous avez raison!, Eh oui! Il a raison!»

Une assertion, positive ou négative, consiste à dire de quelque chose que c'est le cas. Rappelons, dans les termes de la théorie, que la construction de l'existence d'un état de choses est associée à une opération de validation par assignation d'une valeur référentielle: l'occurrence est située par rapport à un système de référence (espace-temps, origine subjective). La validation établit ainsi une relation entre la situation (réelle ou imaginée) et l'une des valeurs possibles.

L'espace de validation est représentable par un domaine topologiquement délimité en deux zones complémentaires - un Intérieur (ce qui est le cas), un Extérieur (vide, absence, altérité) - et une zone de transition ou Frontière":

4. Ce schéma correspond à la construction d'un domaine notionnel, avec les positions définies par la délimitation des zones. L'énoncé «ça c'est un président» nous place dans la zone intérieure, avec une occurrence de la notion 'être-président' conforme au type, alors que l'énoncé «il n'a rien d'un président» nous renvoie à la zone extérieure; «il lui manque l'essentiel pour être un vrai président» nous situerait plutôt à la frontière et «c'est un président du tonnerre, comme c'est pas possible» nous oriente vers ce que l'on appelle l'attracteur, avec une valeur de haut degré. 


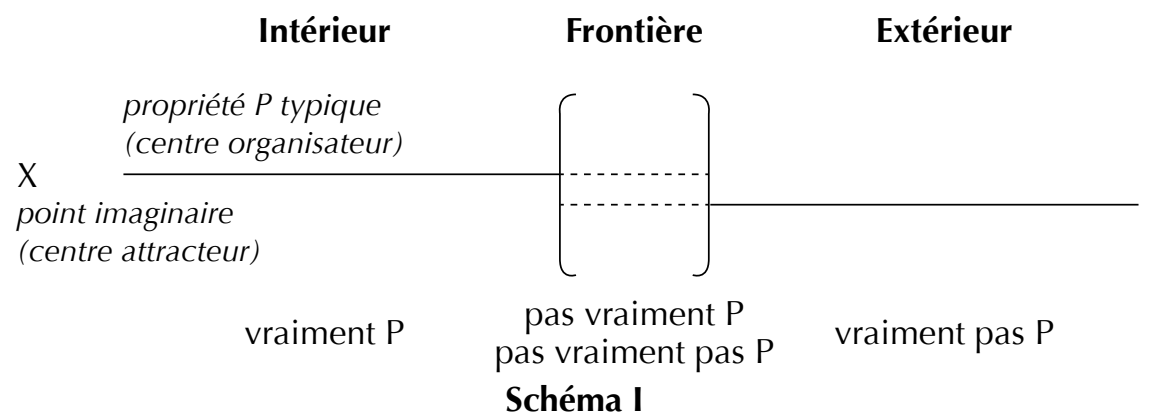

Toute assertion, par le choix d'une valeur, détermine une orientation dans cet espace et implique une position énonciative liée à l'ENGAGEMENT (engagement, désengagement, non-engagement) du sujet énonciateur. Ce point de vue rejoint - si l'on néglige ce qui sépare les deux approches du langage - la définition de la classe des actes assertifs de John R. Searle, dans sa taxinomie des actes illocutoires, quand il présente, de façon générale, l'assertif comme l'une des manières d'employer la langue, en fonction du but illocutoire, en disant à autrui comment sont les choses, et qu'il ajoute que ce but est d'engager, à des degrés divers, la responsabilité du locuteur sur l'existence d'un état de choses ou la vérité de la proposition exprimée (Searle 1982).

Je voudrais m'arrêter à la forme et à l'interprétation du «N'importe quoi ! » qui reprend et (dis)qualifie un propos dans sa prétention à dire ce qui est le cas, et qui peut entraîner, selon une variante prosodique, la sortie de l'échange énonciatif.

\section{Formation et valeurs de n'importe quoi}

Je peux dire qu'il importe, pour la démonstration, de s' arrêter à la forme n'importe quoi, ou encore que cela m'importe de faire venir dans le cours de ce développement des considérations morphosémantiques, en faisant valoir l'importance que je leur accorde. Je situe ainsi la valeur portée à cet aspect linguistique dans le cadre de l'exposé, dans la mesure où cela me semble pertinent pour mon propos.

Ce petit jeu réflexif nous ramène à l'origine latine du verbe (in-, portare: 'porter dans'), d'où procède la signification de 'causer' et 'entraîner', que nous trouvons dans la forme de l'ancien français emporter, attestée jusqu' au XVI ${ }^{\mathrm{e}}$ siècle avec la signification de 'comporter, avoir pour conséquence', comme l'italien importare, 'comporter, impliquer, entraîner' (XIII ${ }^{\mathrm{e}}-\mathrm{XIV}^{\mathrm{e}} \mathrm{s}$.), qui passe ensuite à 'nécessiter, réclamer'. Littré, avec son art du raccourci, résume ainsi l'enchaînement des significations à partir du latin :

«Si l'on suit l'historique, on voit comment "importer" a pris le sens d'être de conséquence: une chose importe, par exemple la conservation de la vie, c'est- 
à-dire elle apporte avec soi...; puis absolument, une chose importe. Dès lors, la transition est faite; et du sens de porter dans ou importer, on touche au sens de avoir de l'importance» (Littré 1969, p. 799).

Ces indications historiques ${ }^{5}$ montrent la relation d'implication construite par le prédicat importer, dans les séquences personnelles (ceci importe) ou impersonnelles (il importe de-, il importe que-). Cette relation peut aussi être qualifiée de relation de pertinence (lat. pertinens: qui concerne), selon le rapport de conséquence existant entre ce qui est le repère qualitatif de la valeur attribuée (le domaine de référence), que le marqueur pour peut introduire, et ce qui est importé dans ce domaine.

La valeur est minorée, tendant vers zéro, quand je dis que cela importe peu, ou annulée par autrui si à ma question - Voulez-vous que je développe ce point?, quelqu' un me répond par un -N'importe, c'est comme vous voulez, en se plaçant dans une position d'indifférence quant aux deux valeurs possibles (développer / ne pas développer). Il n'est pas dit alors qu'iln'importe pas que ce soit développé ou qu'il n'importe pas que ce ne soit pas développé, mais il est dit qu'iln'importe pas que ce soit développé ou non. Dans N'importe, l'adverbe de négation ne, seul, inverse l'orientation et nous ramène à un point IE (Intérieur-Extérieur) correspondant à la frontière du domaine notionnel (voir schéma I) - où les deux valeurs sont équivalentes pour l'énonciateur, la sélection de l'une ou de l'autre étant laissée à l'appréciation de celui qui pose la question ${ }^{6}$.

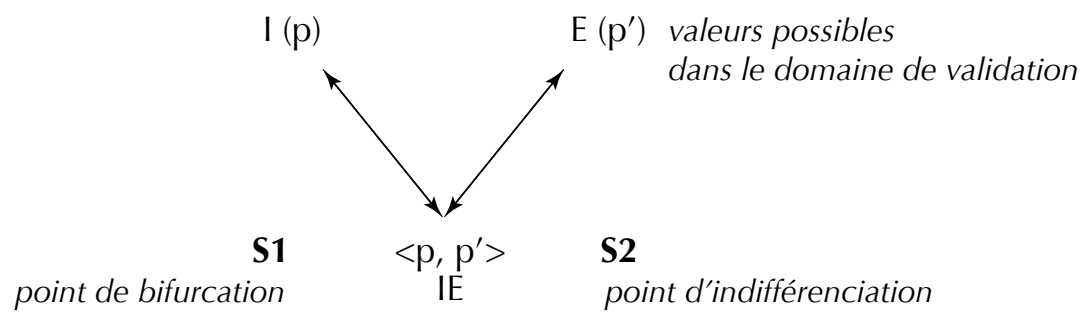

\section{Schéma II}

On pourrait aussi répondre à cette même question par l'exclamative Qu'importe!, renvoyant à la négligence où sont tenues, de la part du sujet, les deux branches de l'alternative: que $\mathrm{P}$ / que non P. La forme expressive peut être glosée en 'ce

5. Voir, pour ces indications, Alain Rey (1992).

6. Le schéma II est une variante du schéma I, avec la valeur p qui représente la zone intérieure I et la valeur p' qui représente la zone extérieure E (non p, autre que p, ou valeur nulle). La frontière IE y est réduite à un point correspondant à une position neutre: absence d'engagement, indifférence, attente (dans l'interrogation par exemple). 
que cela importe est de peu d'importance ou n'a pas d'importance', à quoi il conviendrait d'ajouter 'au regard d'autre chose ou de ce qui concerne le propos': ou encore par 'c'est sans conséquence'. Ces deux dernières expressions (n'importe + groupe nominal et qu'importe + nom / complétive / de v. inf.) sont traditionnellement rangées avec les locutions pronominales indéfinies comme n'importe qui, quoi, lequel, et réunies avec les locutions adjectivées (n'importe quel, quelle) et adverbiales (n'importe comment, où, quand) ${ }^{7}$. Chaque forme d'indéfini suivant la séquence $n$ 'importe, qui ramène au point d'indifférenciation du domaine du validable, renvoie à la classe des valeurs instanciables dont elle est le représentant, dans une relation d'agentivité, d'identification ou de localisation, telle ou telle valeur pouvant servir à valider la relation : le qui / quoi / comment / où / quand, quel qu'il soit, dans la mesure où n'est retenue que l'une des qualités définitoires du domaine dans un espace référentiel donné. Denis Paillard (1997) a proposé une explication des indéfinis «N'importe qui, n’importe quoi, n'importe quel $\mathrm{N} »$ par une oscillation du point de vue selon la prise en compte, ou non, des propriétés singulières de l'individu par rapport à la classe des occurrences instanciables.

Ainsi le bricoleur qui veut colmater une fuite d'eau et qui réclame en urgence quelque chose pour boucher le trou et à qui on demande ce qu'il lui faudrait, peut répondre: «Je ne sais pas, moi. N'importe quoi, mais vite !», étant alors entendu que l'injonction renvoie, en représentation, à tout objet ayant la qualité requise, adéquat à la situation et conforme au type (ce qui est du 'bouchon' ou du 'bouchonnant' pour une fuite d'eau). Les propriétés singulières de l'individu, selon la terminologie de Paillard, sont ici considérées comme évidentes et partagées par autrui. Un énoncé commençant pas «N'importe quoi » peut toujours se poursuivre avec un enchaînement spécifiant la qualité, par restriction conditionnelle : «pourvu que ce soit efficace ». Dans le cas où l'on proposerait à notre bricoleur du papier journal, il pourrait s'exclamer: «N'importe quoi! » (avec une prosodie distincte). Nous sommes passés d'une opération de parcours ${ }^{8}$ sur

7. Les grammaires, qui soulignent le caractère hétéroclite de la catégorie de l'«indéfini», classent les indéfinis (déterminatifs ou pronominaux) en distinguant les identificateurs: $m e ̂ m e(s)$, le même, autre(s), autrui, tel(le)(s) et les quantificateurs, qui sont décrits en référence aux notions de nullité: aucun(e), nul(le), pas un(e), personne, rien, de pluralité: plusieurs, certain(e)s, maint $(e)(s)$, quelques, divers(es), différents(es), quelques-uns(es), de singularité: quelque, n'importe quel, certain, quelqu'un, quelque chose, l'un(e), n'importe qui, n'importe lequel/laquelle, de totalité ou globalité: tout(e), tout le monde, tous (toutes), de distributivité: chaque, chacun(e). La Grammaire méthodique du français (M. Riegel et al. 1994) dit ainsi des pronoms quelqu'un, quelque chose, n'importe qui / quoi et n'importe lequel, qu'ils «fonctionnent, avec des nuances diverses, comme des indicateurs de singularité indéterminée», en ajoutant qu' "Ils admettent tous des compléments partitifs (n'importe qui d'entre nous) et, sauf n'importe lequel, un adjectif épithète introduit par de: quelqu'un / quelque chose /n'importe quoi de beau.» (p. 212)

8. Nous retrouvons l'opération de parcours dans ce que les auteurs de la Grammaire méthodique (Riegel et al.1994) nomment la totalité distributive, à propos de chaque, n'importe quel(le) 
la classe des occurrences qualitatives, avec une équivalence entre les valeurs instanciables dans la relation à valider (boucher un trou avec du bouchon), ce qui est le propre de l'indéfinition, à une valeur extrême et un rejet. Nous pourrions ajouter en commentaire: «c'est tout sauf ce qu'il faut», par exclusion et renvoi à l'extérieur. Ce qui montre que c'est l'ensemble du domaine notionnel (Int., Front., Ext.) qui est représenté et qui est à l'origine des opérations. L'annulation de ce que le second protagoniste a présenté comme du possible fait basculer dans le hors d'état de chose visé («ce n'est vraiment pas à ça à quoi je pensais », pourrait ajouter le premier, ou, en dépliant davantage la glose, «je ne pouvais imaginer que tu penserais que ce serait possible avec ça»). La première occurrence du n'importe quoi porte sur la construction d'un existant possible validable, un quelque chose qui a la qualité-de; la seconde occurrence porte sur cette qualité et invalide la valeur référentielle, par dévaluation'. Nous avons alors une reconfiguration des zones avec de l'existant disqualifié (en tant qu'inadéquat) et une prépondérance de la qualité ${ }^{10}$.

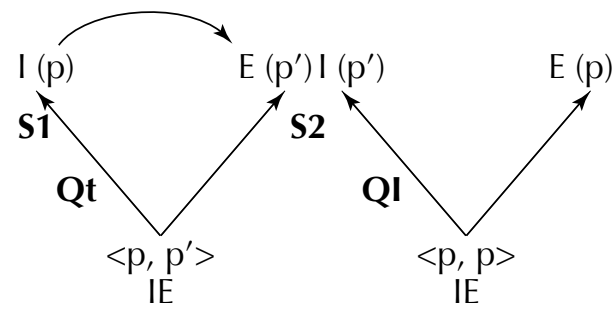

Schéma III

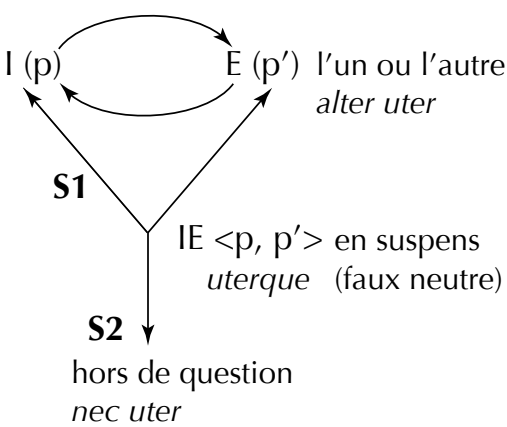

Schéma IV

et tout (e), qui «font référence à la totalité des êtres dénotés par le nom et son expansion, mais en passant en revue séparément les individus constituant cette totalité.» (p. 162)

9. L'on pourrait imaginer ce dialogue entre les deux protagonistes: «Va me chercher quelque chose pour colmater la fuite. - Quoi ? - Je ne sais pas, moi... N'importe quoi, vite! - Tiens, j'ai trouvé ça (en tendant du papier journal) - C'est quoi, ça? Tu te moques de moi. C'est n'importe quoi! - Tu m'as dit de t'apporter n'importe quoi. - Ah ça, pour être n'importe quoi, c'est vraiment n'importe quoi! »

10. Le schéma III illustre le passage d'une valeur à une autre du n'importe quoi dans le petit dialogue imaginé dans la note 9 . Le premier n'importe quoi se réfère à tout existant ayant la qualité requise (ce qui vaut pour boucher le trou), le second renvoie ce qui est proposé à l'extérieur, relativement à cette qualité (c'est vraiment tout autre chose que ce qui convient). Le schéma IV montre l'aller-retour entre les valeurs possibles et la sortie du domaine de validation par disqualification de l'énonciateur, qui dit n'importe quoi. 
Le domaine de qualification du n'importe quoi est construit par l'activité discursive dans l'évaluation d'une mesure d'adéquation entre la notion qualitative représentée par un désignateur et sa valeur référentielle. Je renvoie à l'item 2 (Doc. B en annexe) de l'exemplier, où l'action du gouvernement en matière de financement des $35 \mathrm{~h}$ est qualifiée de «tout et n'importe quoi», selon la notion cohérent / incohérent. L'item 1 (Doc. A en annexe) est intéressant car il pointe le hors de propos dans l'emploi d'une citation dont il est dit qu'elle pourrait s'appliquer à «n'importe quoi », la remarque du commentateur déniant ainsi la valeur référentielle de celle-ci : le propos de la citation, utilisée comme un argument d'autorité morale, n'est pas pertinent dans la situation de référence dans la mesure où son domaine de validation est constitué de tous les cas possibles et imaginables, indistinctement, pour lesquels elle est validable. C'est un critère qualitatif de particularité de la situation, opposé à la généralité de la citation, qui entraîne la disqualification.

La plupart des exemples relevés dans les débats parlementaires sont des interventions ponctuelles, dans un commentaire du discours en cours, sous la forme exclamative «N'importe quoi! », ou ses variantes «C'est (vraiment) n'importe quoi !». La réaction, conséquente à un propos tenu par un énonciateur engagé dans son dit et par son dire, vise à signifier de façon intensive, par la dévaluation, le désengagement (il n'est pas possible de dire ce qui est dit de la façon dont c'est dit à propos de ce qui est dit) et la séparation entre les partenaires du débat. Pour mieux établir le lien entre assertion, validation, engagement et valuation, dans une relation inter-sujets, je vais reprendre, sommairement, les études d'A. Culioli sur lesquelles je m'appuie.

\section{Validation-valuation: engagement et investissement subjectifs}

Voici ce que dit A. Culioli de l'opération de validation inhérente à l'assertion, en mettant en avant la dimension de subjectivité de l'activité énonciative:

«Cette opération de validation (sélection de la valeur estimée adéquate pour un sujet) s'accompagne de deux opérations d'ordre subjectif: (1) engagement du sujet énonciateur qui, par delà son activité de locuteur, tient à dire (rendre accessible à autrui) ce qu'il sait / pense / croit être le descripteur adéquat. D'où soit un marqueur nul ('Il fait beau'), soit les marqueurs de modulation tels que certainement, sûrement, sans doute, naturellement, évidemment, forcément, probablement, vraisemblablement. Le sujet prend en charge ce qu'il dit, grâce à cet engagement modulé, qui va permettre l'ajustement inter-subjectif; (2) valuation par le sujet énonciateur qui assigne une valeur téléonomique (avantageux / désavantageux, efficace / inefficace, etc.) à l'asserté, par rapport aux enchaînements d'énoncés et à la consécution des états de choses (Culioli 2001 : 281). » 
Cette explication se comprend mieux en précisant que l'article d'A. Culioli part d'énoncés tels que «La maison était mal chauffée et inconfortable. Heureusement (qu') il a fait très beau.», où la relation imaginée entre un état de chose effectif ('maison mal chauffée et inconfortable') et un autre, possible, que je dirai ineffectif ('ne pas faire beau'), est 'heureusement' contrariée par la relation entre deux états effectifs. La modalisation, dont l'assertion stricte n'est que le degré zéro, est alors une évaluation qui conjoint la validation et la valuation ${ }^{11}$. Elle implique une distance de l'énonciateur par rapport aux relations établies dans la construction de l'énoncé, distance variable selon que l'on a affaire à du validé, du validable - ce qui est envisagé ou ce qui est imaginé - ou encore du validé mis en suspens. Cette distance peut être annulée, par désubjectivation, mais toute assertion (dans sa forme positive ou négative), dans l'assignation de valeurs référentielles, comporte, en représentation, son avers ('être le cas' / 'ne pas être le cas', ou 'autre qu'être le cas'). À ces valeurs s'ajoutent les valeurs subjectives qui manifestent l'investissement du sujet énonciateur, avec son caractère dual d'énonciateur-coénonciateur.

Dans une situation d'interlocution, par la reprise en écho de ce qui se dit et la chaîne des réactions des locuteurs, se manifeste un jeu de rapprochement ou d'éloignement et un rapport d'orientation convergent ou divergent. Dans l'adhésion et l'assentiment jusqu'à la dissension se traduisent des opérations d'identification, de différenciation ou de rupture caractéristiques de l'ajustement intersubjectif. A. Culioli analyse dans cette perspective la forme exclamative du «Heureusement !» (avec la prosodie), en réaction à la question «Est-ce qu'il sait conduire ce genre d'engin?», exclamation qui pourrait être glosée naturellement par «Encore heureux ! C'est son métier ! Il ne manquerait plus que ça ! Ce serait un comble s'il ne savait pas !». Une étude de ces énoncés montre les chemins tracés par la construction de l'existence fictive d'une valeur possible ('ne pas savoir'), son rejet et le basculement avec un retour sur une valeur renforcée ('savoir').

Le phénomène observé dans ce dernier exemple renvoie à ce qu'A. Culioli a conceptualisé $^{12}$ sous le nom de force assertive (en reprenant la behauptende Kraft de Frege, traduit en anglais par assertoric force), qui désigne la façon dont l'énonciateur déclare en s'engageant ou s'engage dans sa déclaration et est condensée, dans la formule métalinguistique de l'assertion, par je tiens à. La formule complète qui représente le schéma constitutif de l'assertion est Je veux / désire / tiens à parler (écrire) pour dire que je pense/crois / sais que p est le cas. Chacun des prédicats donnés dans cette formule comme des alternatives fait l'objet de distinctions sémantiques, mais il suffira ici de préciser les opérations représentées dans cet agencement. Le je tiens à signale l'engagement du sujet énonciateur, à des degrés variables, avec une force évoluant sur un gradient d'intensité selon

11. Voir sur ce point D. Ducard (2004: 45-57).

12. Voir notamment A. Culioli (1999: 92-99) et A. Culioli (2002). 
la valuation subjective de ce qui est dit, et dont les marqueurs sont discursifs (formes de langue, formes prosodiques et formes posturo-mimo-gestuelles, dont certaines notations graphiques à l'écrit peuvent être l'équivalent). Le prédicat complexe parler (écrire) pour dire indique l'extériorisation matérielle de ce qui est exprimable et communicable (ce qui est visé) dans une formulation orale ou écrite (le dit). La suite des prédicats pense/crois/sais renvoie à une instance de représentation, qui, selon la modalité énonciative de la validation, peut être personnelle (intrasubjective, intersubjective) ou impersonnelle. $p$ est le cas correspond à la référenciation du complexe énonçable de la représentation (p), dont l'occurrence est située dans un espace-temps. Il faudrait aussi faire une place, dans l'énonciation, au que de la formule, dont l'apparente vacuité sémantique (comme avec d'autres mots que l'on nomme parfois «mots vides») participe de la mise à distance dont il a été question.

Dans plusieurs articles $(2001,2002)$ et dans son enseignement oral, A. Culioli montre, par l'analyse minutieuse de marqueurs spécifiques et par la généralisation que cette analyse autorise, comment le sujet énonciateur est pris dans l'imaginaire des représentations qu'il attribue à autrui vis-à-vis de lui-même. C'est notamment le cas quand il réagit à un énoncé en situant ce qui est dit en dehors du domaine de validation des valeurs référentielles et en disqualifiant le coénonciateur. A. Culioli a ainsi analysé l'énoncé «Avec ça ! que j' allais signer », en réponse à la question «Est-ce que tu as signé ?», dont des équivalents seraient: «Moi, signer! tu rigoles! tu n'y penses pas!», et qui pourraient être complétés par des gloses du type «pour qui tu me prends? ! ça va pas la tête! t'es dingue ou quoi ! tu crois (croyais) peut-être que j'allais signer?! qu'est-ce que tu vas imaginer là ?! »? Toutes ces formes de réaction appartiennent à un répertoire fondé sur une catégorisation qui se ramène à une propriété d'ordre agentif: «mettre en question le sérieux de l'autre, en tant que garant d'un énoncé légitime, son bon sens, son statut d'énonciateur (digne de l'idée que l'on se fait de ce que doit être un énonciateur), sa capacité à ne pas bouleverser le jeu des forces symboliques qui organisent le champ inter-sujets ${ }^{13} \gg$.

Le «N'importe quoi! » qui ponctue les débats parlementaires est interprétable comme une disqualification exprimant un désaccord, une désapprobation, un démenti, une dénonciation (comme dans Doc. D, item 5, et Doc. E, item 6, en annexe), mais il reste, sauf dans les situations d'invectives, à la limite du «N'importe quoi !» de rupture énonciative (avec une nuance dans la prosodie:

13. Voir A. Culioli (1999), «Non mais, des fois!», p. 137. Dans cet article, A. Culioli examine l'énoncé «Non mais, des fois!», en soulignant l'ajustement intersubjectif d'ordre téléonomique qui se traduit, dans un espace de confrontation, par un rejet qualifié d'indigné. Il récapitule son analyse en concluant que: «la séquence non mais des fois se construit comme un agencement cohérent de traces d'opérations : rejet du champ intersubjectif de $X$, en tant qu'origine de l'énoncé (non, mais), dont $\mathrm{Y}$ se demande ce qui a bien pu (des fois) amener $\mathrm{X}$ à le produire.» 
débit plus rapide, intonation montante et accent plus fort sur la dernière syllabe), pouvant conduire à l'arrêt de l'interlocution, suite à la disqualification du coénonciateur comme allocutaire valable. L'intensité affective, dont une analyse prosodique devrait rendre compte, et la réaction en chaîne («ça ne va pas la tête ! tu te rends compte de ce que tu dis! pour qui tu me prends!», etc.) traduisent un «N'importe quoi! » qui n'est pas un simple désengagement vis-à-vis du propos soutenu par l'autre, par une sortie de la zone de l'intérieur du domaine de validation pour se placer en dehors, mais un dégagement hors de l'espace de validation, par une remise en cause de l'autre en tant qu'alter ego dans une relation de réciprocité non-symétrique. C'est ce que j'ai nommé, dans le titre, le hors-sujet de l'énonciation, en jouant sur le lien entre le hors de propos ou de question et le dégagement du sujet énonciateur. Nous pouvons donner la glose métalinguistique suivante, avec un énonciateur $X$ et un coénonciateur $Y$ : tu ne crois / imagines quand même pas que $\mathrm{p}$ est le cas $\mathrm{p}$ étant la représentation par $\mathrm{X}$, consécutif à un énoncé de $\mathrm{Y}$, de la représentation attribuée à $\mathrm{Y}$ de ce que $\mathrm{X}$ dit / fait / pense.

Il s'agit d'un jeu d'images déclenché par une interprétation. Michel Pêcheux avait essayé, en son temps, de formaliser l'intrication de ce qu'il nommait des «formations imaginaires» dans l'activité discursive, qui fait de la communication autre chose que de la transmission d'information ${ }^{14}$.

L'hypothèse que j'avancerai ici est que ce double versant des relations, depuis la construction de l'existence jusqu'à la valuation dans une relation intersubjective, c'est-à-dire distance entre la représentation et la référenciation (ce qui est pensé dans ce qui est dit et ce à quoi il est référé en le disant), distance entre les valeurs possibles (ce qui est dit par rapport à ce qui n'est pas dit), distance dans la relation énonciateur-coénonciateur (le moi etl'autre, avec toutes ses variantes), manifeste, de façon diverse, dans les échanges discursifs, l'écart symbolique qui est la condition de l'activité de langage. Dans cet écart ${ }^{15}$, le sujet, du point de vue des opérations énonciatives, s'engage par la validation, s'investit par la valuation. J'ajoute donc à la notion d'ENGAGEMENT celle d'investissement, avec la signification qu'elle a en psychanalyse de désigner la valeur affective attachée à une représentation. Pour nous en tenir à une approche sémio-linguistique, rappelons que le dernier séminaire de Roland Barthes s'intitulait «Qu'est-ce que

14. Voir M. Pêcheux (1969). Les «formations imaginaires» désignent «la place que A et B [le destinateur et le destinataire du schéma de la communication] s'attribuent chacun à soi et à l'autre, l'image qu'ils se font de leur propre place et de l'image de l'autre» (p. 19).

15. La notion d'écart, anciennement «entaille, incision» $\left(12^{\mathrm{e}} \mathrm{s}\right.$. selon le Robert historique, Rey 1992), est à comprendre, selon la question à traiter, dans les différentes acceptions que donne Le Petit Robert: distance qui sépare deux choses qu'on écarte ou qui s'écartent l'une de l'autre; différence entre deux grandeurs ou valeurs; action de s'écarter, de s'éloigner d'une direction ou d'une position; ou encore, comme en balistique, distance séparant le point de chute d'une trajectoire d'un point idéal dit «point moyen de tir». 
"tenir un discours"? Recherche sur la parole investie» (Barthes 2002). Partant des impressions de tension et de mobilité suscitées par le développement d'un discours de Charlus au narrateur dans Du côté de Guermantes ${ }^{16}$. Il déclarait qu'il convenait de concevoir le discours «comme un jeu de forces, d'intensités mobiles (idée de la moire, des déclencheurs, des "tactèmes", des "infléxèmes") ». Il en appelait alors à une sémiologie qui ne serait plus seulement une sémiologie des places et des forces mais aussi une sémiologie des intensités. Ce serait une sémiologie de la parole vive, faisant le lien entre le corps et le discours. La notion de force assertive, associée à l'engagement et à l'investissement, y contribue.

\section{Références}

BARthes, R., 2002, Comment vivre ensemble. Simulations romanesques de quelques espaces quotidiens. Notes de cours au Collège de France (19761977), Texte établi, annoté et présenté par Claude Coste, Paris, Seuil.

Culioli, A, 1970, «Modalité», in Encyclopédie Alpha, t.10, Paris, Grange Batelière.

Culioli, A., 1985, Notes du séminaire de DEA 1983-1984, Université Paris 7 , Département de recherches linguistiques, Poitiers.

Culiol, A., 1999, «Non mais, des fois!», in Pour une linguistique de l'énonciation. Domaine notionnel, t. 3, Paris, HDL, Ophrys, pp. 135-141.

Culioli, A., 1999, «Accès et obstacles dans l'ajustement intersubjectif», in Culioli, A., 1999, Pour une linguistique de l'énonciation. Domaine notionnel, t. 3, Paris, Ophrys.

Culioli, A., 2001, «Heureusement!», in Correia, C. N. \& Mira Mateus, M. H. (éds), 2001, Saberes no tempo. Homenagem a Maria Henriquez Costa Campos, Lisboa, Edições Colibri, pp. 279-284.

Culioli, A., 2002, «JE VEUX! Réflexions sur la force assertive», in Botella, C. (éd.) 2002, Penser les limites. Écrits en l'honneur d'André Green, Paris, Delachaux et Niestlé, pp. 102-108.

DuCARD, D., 2004, «Formes de discours et énonciation: la modalisation», in DuCARD, D. (éd.), 2004, Entre grammaire et sens, Études sémiologiques et linguistiques, Paris, HDL, Ophrys, pp. 45-57.

LitTRE, E., 1969, Dictionnaire de la langue française [1863-1872], tome 4, Paris, Gallimard-Hachette.

Paillard, D., 1997, «N'importe qui, n'importe quoi, n'importe quel N », Langue française, 116, pp. 100-114.

PÊCHeux, M., 1969, Analyse automatique du discours, Dunod, Paris.

REY, A. 1992, Dictionnaire historique de la langue française, Paris, Dictionnaires Le Robert.

16. Proust, Le côté de Guermantes, II, chap. II, Paris, Gallimard, Pléiade, pp. 553-561. 
Rey-Debove, J. \& Rey, A., 1995, Nouveau Petit Robert, Paris, Dictionnaires Le Robert.

Riegel, M., Pellat, J. C. \& Rioul, R., 1994, Grammaire méthodique du français, Paris, PUF.

SEARLE, J. R., 1982, Sens et expression. Études de théorie des actes du langage [1979], Paris, Minuit. 


\section{Annexes}

\section{ASSEMBLÉE NATIONALE DÉBATS PARLEMENTAIRES \\ Journal officiel de la République française Comptes rendus intégraux ${ }^{17}$}

\section{DOC. A}

[AN, DP, JO 27 nov. 2002, séance du 26 nov. 2003. Vote et éligibilité des étrangers aux élections locales. Discussion d'une proposition de loi constitutionnelle]

\section{ITEM 1}

M. Bernard Roman, rapporteur. [...]

Le principe d'égalité auquel se réfère notre République ne s'accommode pas de la moindre discrimination! Nous nous honorerions en l'admettant, en faisant nôtre cet écrit d'Albert Camus dans ses Carnets: «Si l'homme échoue à concilier la justice et la liberté, alors il échoue à tout.» (Applaudissements sur les bancs du groupe socialiste et du groupe des député-e-s communistes et républicains. - Exclamations sur les bancs du groupe de l'Union pour la majorité présidentielle. M. Thierry Mariani. Vous avez échoué longtemps et cela va continuer!

M. Manuel Valls. M. Mariani est convaincu !

M. Claude Goasguen. Vous avez consulté le Dictionnaire des citations à la rubrique «Camus», monsieur Roman? Cette citation peut s'appliquer à n'importe quoi !

M.André Gerin. Vous, monsieur Goasguen, vous mériteriez de figurer dans le Guiness des records.

\section{DOC. B}

[AN, DP, JO du 21 oct. 2003, séance du 20 oct. 2003, Loi des finances 2004. Discussion d'un projet de loi]

\section{ITEM 2}

M. Charles de Courson. [...]

Quant aux incroyables modalités de financement des 35 heures, on a vu tout et n'importe quoi : du tabac, de l'alcool, des taxes sur les assurances, des taxes sur les véhicules, la TGAP, et j'en passe. C'est complètement aberrant. Il est donc bon que le Gouvernement le supprime et qu'il redistribue les différentes recettes d'une façon un peu plus cohérente, car, même si l'on peut discuter de certains points, c'est sans commune mesure avec ce que vous avez fait.

Il faut donc voter cet article.

DOC. C

|AN, DP, JO du 24 juin 2003, séance du 23 juin: Réforme des retraites. Discussion des articles du projet de loi]

\section{ITEM 3}

M. Jean-Pierre Brard. La pédagogie, monsieur Accoyer! Vous, vous êtes médecin, moi je suis instituteur! Nous ne sommes donc pas dans la même discipline. Et vous savez, quand on est ins-

17. Source : http://www.journal-officiel.gouv.fr/ 
tituteur, le problème, c'est toujours de s'exprimer pour être compris et pour essayer de transmettre le savoir aux enfants qui nous sont confiés.

Alors, que dit M. Fourgous? C'est très intéressant. Tout à l'heure, M. Fillon nous disait: je vais vous éviter de faire une bêtise. Mais, là, évidemment, M. Fillon ne peut pas vous suivre, monsieur Fourgous, parce que vous affichez trop clairement quelles sont vos intentions. Mais en même temps, comme dans toute chose inexacte, il y a un fonds de vérité dans votre amendement.

Que dit M. Fourgous? Dans l'exposé sommaire, alors qu'il parle de travailleurs qui sont usés, ceux-là mêmes dont je parlais tout à l'heure, qui n'en peuvent plus - je pense aux éboueurs, aux ouvriers de la voirie, à bien d'autres encore -, il dit ceci : «En cas de départ volontaire, à partir de soixante ans, ces salariés ne recevraient que l'indemnité de départ à la retraite, très inférieure, généralement, à l'indemnité de mise à la retraite. » Vous voyez, tout est dit. Et, monsieur le ministre, je comprends que vous ayez interdit aux députés UMP de parler et que seul Denis Jacquat soit investi de votre confiance (Protestations sur les bancs du groupe de l'Union pour un mouvement populaire),...

M. Denis Jacquat. Nous avons l'art de la synthèse!

M. Jean-Paul Garraud. Hors sujet!

M. Jean-Pierre Brard. ... parce que dès qu'ils disent quelque chose, cela renforce le point de vue que nous défendons. Alors, je reconnais que M. Fourgous était sincère,,..

M. Jean-Paul Garraud. Il ne dit pas n'importe quoi, c'est tout.

M. Jean-Pierre Brard. Ça, pour dire n'importe quoi, il y en a sur qui on peut compter, justement ! Enfin écoutez, mon cher collègue, si on vous interdit de parler, du côté de l'UMP, il doit bien y avoir une raison!

M. Jean-Paul Garraud. Personne ne m'interdit de parler!

M. Jean-Pierre Brard. C'est pour éviter de dire des bêtises, comme dirait M. Fillon! M. Fourgous, lui, a eu le courage de parler, et il a dit la vérité. Alors, il retire son amendement sous votre amicale pression, monsieur le ministre: évidemment, c'est parce qu'il dévoile ce qu'est le fond de votre texte.

M. Eric Raoult. Vous ne parlez pas, vous provoquez!

\section{ITEM 4}

M. le président. La parole est à M. Jean-Pierre Brard, pour répondre à la commission.

M. Jean-Pierre Brard.

[...] Mais il est un aspect de la démographie dont vous ne parlez jamais, et je le comprends puisqu'il dessert votre argumentation, c'est que la natalité est excellente en ce moment chez nous!

M. Pierre Lellouche. N'importe quoi! D'où sortez-vous çà?

M. Jean-Pierre Brard. Je vous renvoie à l'INSEE et aux statistiques européennes.

En descendance finale, nous sommes à 2,18 enfants par femme, juste après l'Irlande.

M. Bernard Accoyer, rapporteur. C'est 1,85!

M. Jean-Pierre Brard. Et les études démontrent que si la demande d'équipement pour la petite enfance était satisfaite, les couples auraient un nombre d'enfants qui permettrait une descendance finale de 2,35 à 2,40.

M. Pierre Lellouche. Mais non!

M. Jean-Pierre Brard. Voilà de quoi financer les retraites à l'horizon 2040 !

M. Bernard Accoyer, rapporteur. C'est faux!

M. Jean-Pierre Brard. Monsieur Lellouche, qui veut noyer son chien l'accuse de la rage. Vous ne voulez pas voir ces horribles statistiques. Or la reprise de la natalité est bien réelle dans notre pays depuis 1997-1998. Que cela bouscule vos plans et contrarie le discours fantasmatique que vous développez pour faire avaler la pilule amère à nos compatriotes, c'est certain. Que vous développiez ce discours, c'est logique, mais admettez au moins que vous racontez, comme on dit dans ma Normandie natale qui est proche de la Sarthe de M. Fillon, des «menteries»! 


\section{DOC. D}

[AN, DP, JO du 9 juillet 2004, séance du 8 juillet 2004: Maîtrise de l'immigration et séjour des étrangers en France. Discussion des articles d'un projet de loi]

\section{ITEM 5}

M. Serge Blisko. On voit bien ici la logique un peu folle dans laquelle nous sommes entrés cet après-midi avec cette histoire de certificats d'hébergement. On apprend maintenant qu'il y a un fichier des hébergeants. Il y aura ceux qui en auront trop fait, trop demandé, avec qui on n'acceptera pas parce qu'on a déjà refusé une fois. Enfin bref, on entre dans une logique totalement folle. M. Pierre Cardo. Qu'y a-t-il de si fou?

M. Serge Blisko. Je voudrais revenir un instant au débat sur l'espèce de dépôt de garantie financière. Dans la loi française, avant d'être condamné, il faut quand même qu'il soit prouvé qu'on est coupable. Prenons un cas qui peut arriver, et qui doit même arriver tous les jours: vous hébergez votre cousine chez vous - je ne regarde personne en particulier. Vous déposez de l'argent - je ne sais pas où, à la Caisse des dépôts ou ailleurs. Elle vous a dit qu'elle partirait au bout d'un mois. Elle ne part pas. Vous allez être sanctionné et, en plus, être obligé de l'accompagner à l'aéroport, de vérifier qu'elle a bien franchi le portillon? Il y a là quelque chose de totalement inapplicable. Vous allez être condamné parce que, de bonne foi, vous avez cru que votre cousine allait partir alors qu'elle est restée ? Et on va vous inscrire sur un fichier? Quand nous disions ce matin qu'on entrait dans une espèce de logique policière,...

\section{Claude Goasguen. N'importe q uoi !}

M. Serge Blisko. ... totalement inapplicable, et contraire à la Constitution, je crois que nous étions en deçà de la vérité, qui se révèle cet après-midi.

M. Claude Goasguen. C'est le contraire !

DOC. E

[AN, DP, JO du 28 juillet 2004, séance du 27 janvier 2004. Questions au gouvernement]

\section{ITEM 6}

M. François Brottes. Pardonnez-moi de me montrer ainsi irrévérencieux, monsieur le ministre mais, très honnêtement, je ne peux pas vous laisser dire que nous sommes en train de prendre des dispositions destinées à la réappropriation du territoire par le tissu sanitaire.

Mille exemples vont à l'encontre de ce propos, mais je n'en citerai qu'un: trois hôpitaux de ma circonscription vont être délocalisés. Pour l'un d'entre eux, le financement de l'opération sera assuré dans le cadre du plan Hôpital 2007. C'est aussi le seul qui n'est pas situé dans un couloir d'avalanche. Je vous ai demandé voilà bientôt cinq mois la raison de cette décision. Je n'ai toujours pas reçu de réponse.

D'autres exemples relatifs à l'aménagement du territoire ont été susurrés dans mon dos. De grâce, essayons de ne pas raconter dans l'hémicycle autre chose que ce qui se passe sur le terrain!

Deuxième remarque: nous sommes en train d'inventer une nouvelle forme de féodalité. Demain, les maires des communes rurales rendront la justice sous un chêne et lèveront leur propre armée. Ils devront en particulier recruter un adjoint à la santé.

M. Jean Auclair. Quelle caricature! C'est ridicule!

\section{Richard Mallié. N'importe quoi !}

